\title{
Un procedimiento para evaluar el riesgo de la innovación en la gestión del mantenimiento industrial
}

\author{
A procedure for assessing the risks of innovation in the management \\ of industrial maintenance
}

\author{
Fernando F. Espinosa ${ }^{1} \quad$ Acires Dias $^{2} \quad$ Gonzalo E. Salinas ${ }^{1}$ \\ Recibido 15 de marzo de 2010, aceptado 16 de mayo de 2012 \\ Received: March 15, 2010 Accepted: May 16, 2012
}

\begin{abstract}
RESUMEN
Se presenta un estudio sobre las posibles causas de riesgo en un proceso de innovación de la gestión del mantenimiento industrial, las cuales podrían llevar a que la implementación de las mejorías en la gestión no entregue el resultado esperado. Se describe el proceso de innovación y se identifican las fuentes de riesgos en el desarrollo del proyecto de innovación, finalizando con una matriz de valoración y jerarquización de las acciones que minimizan la posibilidad de que el riesgo sea realidad.

Palabras clave: Gestión del riesgo, gestión del mantenimiento, optimización, innovación, estrategias.

\section{ABSTRACT}

This study shows the possible causes of risk in a management innovation process of the industrial maintenance, which could take to that the improvements implementation in the management does not give the expected result. The innovation process is described and the risks sources in the innovation project are identified finalizing with an evaluation matrix and hierarchical classify for the actions that diminish the possibility that the risk will be certainty.
\end{abstract}

Keywords: Risk management, maintenance management, optimization, innovation, strategies.

\section{INTRODUCCIÓN}

Enfrentar un proceso de innovación en la forma de hacer la gestión del mantenimiento trae consigo riesgos asociados a las múltiples tareas que hay que llevar a cabo, que emergerán durante la etapa de levantamiento de los requerimientos para atender los objetivos de negocios de la empresa hasta el último paso que es la valoración de los beneficios que traerá consigo la innovación propuesta. Las incertezas pueden acompañar el proceso en el momento de seleccionar el modelo de concepción más adecuado, cuando se define al líder del proceso de cambios, en poseer la información adecuada, entre otros tantos aspectos.
Las fallas en un proyecto de innovación son el resultado de la multiplicidad de riesgos inherentes en el proceso de desarrollo del proyecto, porque se trata de un conjunto de etapas con muchas interacciones y dependencias entre ellas, además que involucra la creación de algún producto o servicio que nunca antes fue hecho, aunque el proceso de desarrollo sea similar con otros proyectos.

La identificación del riesgo es la fase inicial para su gerenciamiento y es un proceso que revela y determina los riesgos posibles que podría enfrentar el equipo de trabajo de la organización. La identificación se realiza por la investigación de las actividades organizacionales en todos los

1 Universidad de Talca. 2 Norte 685. Talca, Chile. E-mail: fespinos@utalca.cl; gsalinas@utalca.cl

2 Universidade Federal de Santa Catarina. Brasil. E-mail: acires@emc.ufsc.br 
sentidos y en todos los niveles administrativos y es la base para todo trabajo futuro correctamente hecho en la organización [1].

Para aumentar las posibilidades de éxito de un sistema innovador es necesario en la organización tener una comprensión del riesgo potencial, evaluar sistemática y cuantitativamente estos riesgos, anticipando las causas y efectos posibles, y escoger entonces los métodos más apropiados para tratarlos. Hay metodologías y técnicas que pueden ser utilizadas para esta actividad como las presentadas en referencias [2-5]. En este trabajo se presenta el desarrollo de una metodología que va en ayuda del grupo de analistas a cargo de evaluar el proceso de innovación.

Una vez identificados estos riesgos pueden ser reducidos, removidos, evitados o aceptados. La organización necesita también adoptar una actitud más proactiva hacia el riesgo, comprendiendo cómo una evaluación y un análisis eficaz pueden ser usados para anticiparse a los riesgos potenciales al diseñar o ejecutar sistemas nuevos, y minimizar de ese modo aquellos riesgos [6].

Para asegurarse de que todos los riesgos potenciales estén controlados eficazmente, el proceso de análisis del riesgo necesita ser construido explícitamente en el proceso de toma de decisiones. Un análisis del riesgo puede ser resumido en tres etapas: (1) identificación: todos los riesgos potenciales que afectan un proyecto están identificados; (2) estimación: están determinados los riesgos y evaluados con su importancia, su probabilidad, su severidad y su impacto; y (3) análisis y evaluación: están evaluadas la aceptabilidad del riesgo determinado y las acciones que pueden hacer el riesgo más aceptable.

\section{EL PROCESO DE INNOVACIÓN}

El proceso de innovación de la gestión comienza con la definición de la misión de la función mantenimiento conforme con las estrategias y tácticas de la empresa definidas para alcanzar sus objetivos de negocios. Esta es la primera información que deberá tener la función mantenimiento para dimensionar su meta, la cual debe ser totalmente compatible con la de la empresa. El proceso completo es descrito en la Figura 1.
En la etapa de evaluación del nivel de atención de los equipamientos productivos, mantenimiento, producción y logística a los objetivos de la empresa se explora la condición de los equipamientos para atender dichos objetivos, conjuntamente con los servicios relacionados: mantenimiento, operación, abastecimiento (logística) y capacidad administrativa.

La evaluación del nivel de atención de los requisitos es calificada conforme a la visión del equipo que atiende la función mantenimiento. El objetivo es tener una visión bastante clara del nivel de atención de los equipamientos, a esos requisitos, como también de los servicios que la empresa tiene y que están relacionados con el parque de máquinas.

Otro análisis realizado es el de la situación actual de la función mantenimiento y esta parte está orientada a evaluar a la función en relación a la gestión de los aspectos humanos, técnicos y económicos. $\mathrm{La}$ situación es analizada bajo el punto de vista de la capacidad administrativa del responsable del mantenimiento y los resultados pueden ser usados para analizar el estado actual que la organización tiene en relación al sistema de administración, métodos y procedimientos, a la estructura organizacional, al sistema de información y al uso de tecnología para el mantenimiento.

Como en todos los aspectos considerados, la interpretación de los resultados (la apreciación del nivel de desarrollo) es una instancia muy particular del administrador o del encargado del mantenimiento. De cierta forma, está al alcance de él la percepción de la capacidad de administración y del nivel de respuesta de sus recursos en relación a los objetivos fijados para la función mantenimiento.

Con respecto al análisis de la madurez de la organización se aplica este concepto en la organización para constatar el estado donde la organización está en relación a los atributos que están siendo evaluados [7]. La madurez de la función mantenimiento podría indicar que la organización definida para la mantenimiento está perfectamente condicionada para emprender todos sus proyectos de innovación, sean tecnológicos o de gestión integral de sus recursos.

La medición de la madurez es más subjetiva que objetiva y depende del juicio del administrador 


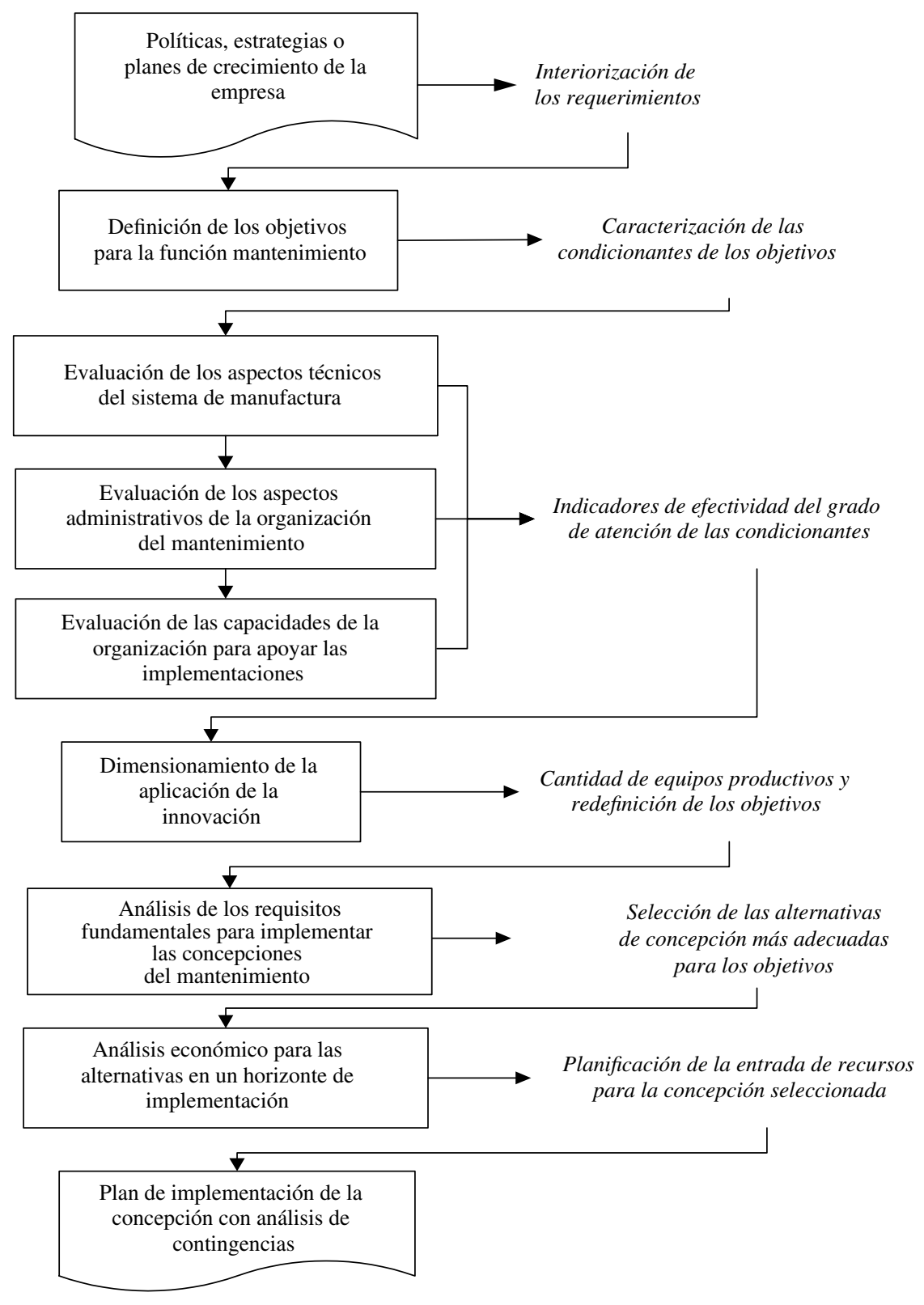

Figura 1. Proceso de análisis para la innovación de la gestión del mantenimiento.

sobre su entorno. La madurez es principalmente, la combinación del apoyo o comprometimiento que el entorno ofrece a la función mantenimiento más la capacidad o conocimiento que se tiene de las metodologías relacionadas con implementación de proyectos [8].

En el análisis de la criticidad de los equipamientos y de la función mantenimiento el objetivo es decidir si el proceso de innovación se aplica a toda la línea o solamente a un grupo más reducido de equipamientos y si se aplica también la innovación a todas las funciones que involucra la gestión del mantenimiento. Es recomendable no abarcar todo el conjunto de equipamientos, en especial si este conjunto fuese numeroso y de alta complejidad. Es preferible concentrarse en adquirir experiencia en equipamientos que son conocidos y para los cuales 
con la introducción de mejoramientos, sean estos tecnológicos o de gestión, se obtienen resultados apreciables. También se define el número de equipamientos que entrarán en la primera etapa del estudio, lo que influye directamente en el análisis de costos para cada concepción.

El análisis de los parámetros y selección de la concepción del mantenimiento está referido a la selección de aquellos parámetros que definen las características de cada concepción y que están relacionados con los aspectos técnicos, administrativos, de gestión, de apoyo del entorno, de conocimientos básicos, de uso de metodologías y herramientas de optimización y de modelos de proyecto. Para cada uno de ellos se fija un nivel mínimo que la función mantenimiento debería atender en conformidad con su entorno competitivo y el mercado que enfrenta y estos niveles son comparados con las evaluaciones ya hechas en las etapas anteriores. Se tiene un punto de referencia sobre las condiciones cualitativas que la organización posee para la implementación de algunas concepciones.

Finalmente se llega al análisis económico de las alternativas de mantenimiento y es un paso importante en la selección final de la concepción del mantenimiento, ya que en esta parte se valora el real impacto de la innovación en la gestión. Este módulo es el más difícil de realizar, porque cuantificar los beneficios es una tarea que requiere un conocimiento ( $\mathrm{y}$ una estimación del futuro) que muchas veces no está presente, en especial cuando se está trabajando delante de una experiencia innovadora y su valoración requiere el trabajo de un equipo experimentado y calificado.

\section{LAS FUENTES DE RIESGOS Y SU IDENTIFICACIÓN}

Según [9] la forma más adecuada de abordaje para conseguir una buena decisión es considerar la toma de decisiones como un proceso de análisis, considerando un riesgo formal para proveer apoyo, seguido por un juzgamiento informal del administrador y un proceso de revisión, resultando finalmente en una decisión, tal como se muestra en la Figura 2.

El proceso de análisis es usado para evaluar, de forma completa, los variados aspectos que están relacionados con la función mantenimiento e identificar los más importantes que deben ser mejorados en el sentido de direccionar correctamente el proceso de innovación de la gestión del mantenimiento. Hay que tener presente que en la cadena de requisitos, aquel que es considerado deficiente tiende a marcar el ritmo del proceso de gestión. En otras palabras, es más productivo eliminar las debilidades detectadas en el proceso de evaluación del sistema antes de seguir para el próximo paso en la implementación de la concepción, de lo que reconsiderar cuando el proceso ya estuviese encaminado para resolver estos problemas que quedaron pendientes.

En cada etapa de la aplicación de un modelo de evaluación se genera un conjunto importante de informaciones sobre los puntos fuertes y débiles de la gestión actual y aspectos que pueden ser potenciados en la organización del mantenimiento. Estos análisis entregan información para tener un lazo de retroalimentación para definir planes de mejoramiento, eliminar los factores negativos y volver a la implementación de la concepción con

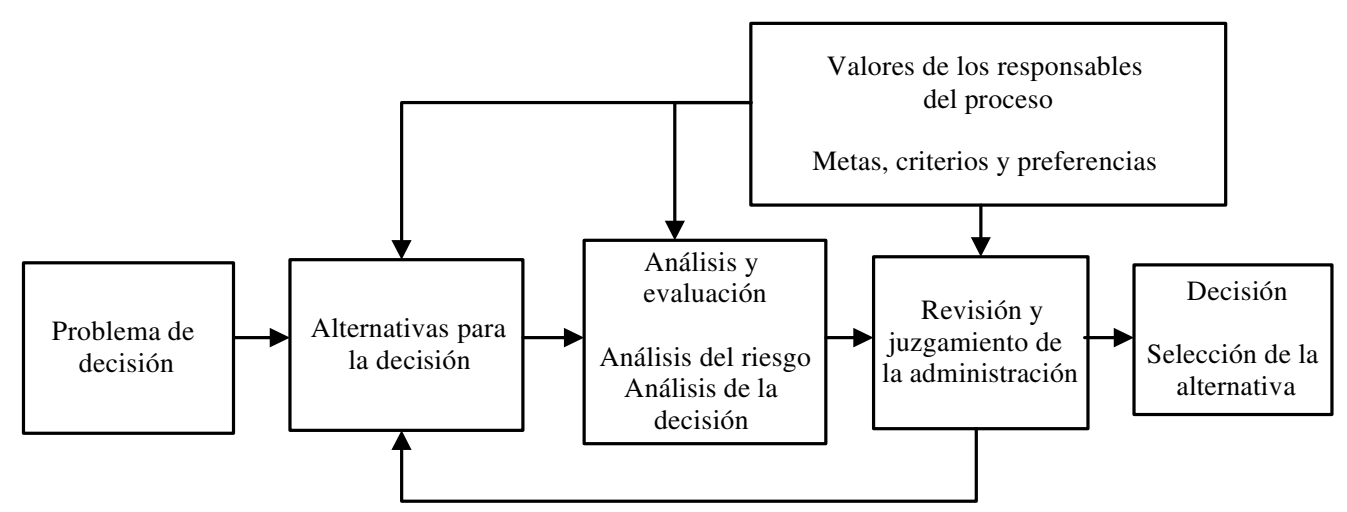

Figura 2. Proceso de análisis y evaluación para la toma de decisiones con riesgo inherente. 
una base más robusta para la gestión, lo que dará a la función mantenimiento las herramientas para alcanzar sus objetivos.

Es cierto que todo el proceso de evaluación está apoyado principalmente en escalas de valoración, muy sujetas a la apreciación del encargado del mantenimiento. Así, para evitar las distorsiones, producto de la apreciación cualitativa, el equipo de analistas debe ser siempre el mismo en todo el estudio.

Las transformaciones organizacionales han ayudado a algunas compañías a adaptarse a los cambios de las condiciones en sus ambientes, a obtener un incremento en la competitividad y a posicionar compañías para un futuro mejor. Sin embargo, en diversas situaciones, los resultados han sido decepcionantes, implicando desperdicio de recursos, pérdida de confianza en los agentes de cambio, frustración en las personas involucradas y, tal vez lo peor de todo, miedo de enfrentar nuevos cambios [10]. Esos fracasos ocurren porque los administradores tienen gran dificultad en identificar, priorizar y alinear apropiadamente sus recursos para hacer frente a los muchos factores que producen transformaciones organizacionales.

Diversas preguntas vienen a la mente durante cualquier proceso de cambio organizacional o funcional [10], las cuales deben ser tratadas con el máximo cuidado en el momento de la implementación de un proyecto de innovación:

- ¿Será que existe un claro motivo para ese cambio? ¿Será que ese motivo es comprendido por las personas del equipo de mantenimiento, de producción y por las personas claves de la empresa?

- Antes de eso, ¿será que existe una clara visión de la situación de la empresa compartida por los individuos de forma de existir consenso sobre lo que debe ser cambiado?

- ¿Quién va a ser el agente (o agentes) que va a conducir el proceso de cambios? ¿Cuáles deben ser las características de ese agente?

- ¿En qué nivel será conducida la construcción de las alternativas de cambio? ¿El nivel superior de la función? ¿Las personas del nivel operacional deberían estar involucradas?
Otros aspectos que también son fuentes de riesgo y se relacionan con los requerimientos de tecnología, conocimientos, capacidad de administración y disponibilidad de recursos, se pueden expresar como:

- ¿Los equipos productivos atienden la evolución tecnológica, estructural y operativa de los productos fabricados? ¿Contribuyen en la reducción de costos de producción? ¿Pueden ser intervenidos a fin de aumentar la productividad, mejorar la calidad, disminuir el consumo de energía, ser más seguros en su operación y disminuir la polución?

- ¿Cómo se planean, organizan, analizan y controlan los servicios, estructura, calidad, métodos de trabajo, recursos y materiales de mantenimiento? ¿Se mide la economía del mantenimiento?

- ¿Cómo manifestar las metas de mantenimiento: mantener la capacidad de utilización, reducir los trabajos de mantenimiento, aumentar la confiabilidad de la línea, mejorar la mantenibilidad, mejorar la seguridad, aumentar la disponibilidad?

- ¿Se está en condiciones de evaluar económicamente la producción rechazada, producción perdida por fallas imprevistas, tiempos de preparación, ajustes o disminución de velocidad de producción, el exceso de inventarios y productos intermedios, perdidas por entregas atrasadas o faltantes, pérdidas por deterioro de los equipos?

- ¿Se tiene la preparación para identificar los requerimientos de información y conocimientos, de técnicas y herramientas, de infraestructura y de apoyo logístico para innovar la gestión?

- ¿Existe la capacidad para evaluar los beneficios y costos en un proyecto de inversión para implementar una nueva concepción del mantenimiento?

El primer grupo de cuestionamientos es importante ya que sus respuestas tienen que ser convincentes y esto requiere de un estudio profundo de las condiciones actuales del mantenimiento, sus deficiencias y fuerzas, una evaluación de su posición relativa con respecto de las empresas competidoras, en la forma como el mantenimiento apoya a la empresa y contribuye positivamente para su éxito. No es suficiente que el administrador esté seguro de la necesidad de un cambio, los motivos tienen que ser demostrados con 
cifras y hechos para que todos los niveles jerárquicos de la organización apoyen con nuevas ideas y den las facilidades para que el equipo que liderará los cambios tenga éxito.

Aquí ya adquiere relevancia el dominio por parte del administrador de herramientas cuantitativas para apoyar su proceso de evaluación de las bondades del proyecto.

El segundo grupo de preguntas son igualmente importantes y están relacionadas con los aspectos del conocimiento de técnicas y herramientas, disponibilidad de recursos, capacidad de trabajar en equipo, facilidad para administrar los cambios y los conflictos que de ellos derivan, sistema de evaluación de la efectividad de los cambios y buena relación con los niveles superiores.

En la Figura 3 se entrega una lista, no siendo esta exhaustiva, de factores presentes en el proceso de análisis, que por sus características de información en primer lugar, y de manejo de relaciones humanas en segundo lugar, son fuentes de riesgos para el buen final del proyecto.

Así, para cada estudio específico que hay que realizar en la evaluación de la factibilidad de implementar una innovación en el proceso de gestión, se pueden identificar aquellos factores que son parte integrante de cada etapa en el desarrollo, y para los cuales el equipo de mantenimiento debe procurar la información más actualizada y sus relaciones con otros sistemas y personas, tal que refleje el real comportamiento del sistema bajo estudio, y de esta forma generar planes de contingencia para el caso que se presenten situaciones no deseadas, o bien, generar con anticipación las condiciones para tener un proceso de innovación con alta probabilidad de éxito.

Los factores de riesgo a identificar por el equipo de estudio pueden ir desde la compatibilización de los objetivos de la empresa con los de mantenimiento y expresarlos en términos de parámetros medibles mediante los resultados de la gestión, hasta la cuantificación de los costos y beneficios de la futura implementación de una concepción del mantenimiento.

Otros factores que pueden afectar el análisis, según [12] incluyen: una especificación vaga de los requisitos, la ausencia de un análisis preliminar de los riesgos, una documentación incompleta del análisis efectuado, complejidad de las interrelaciones y número de influencias entre factores, mucho tiempo asignado al análisis y no respetar las características propias del personal involucrado en el análisis. Para lo anterior, hay que tener presente que un estudio de riesgos precisa de una cuidadosa preparación, asegurando un abordaje sistemático con objetivos y metas claras, el usuario necesita tener suficiente

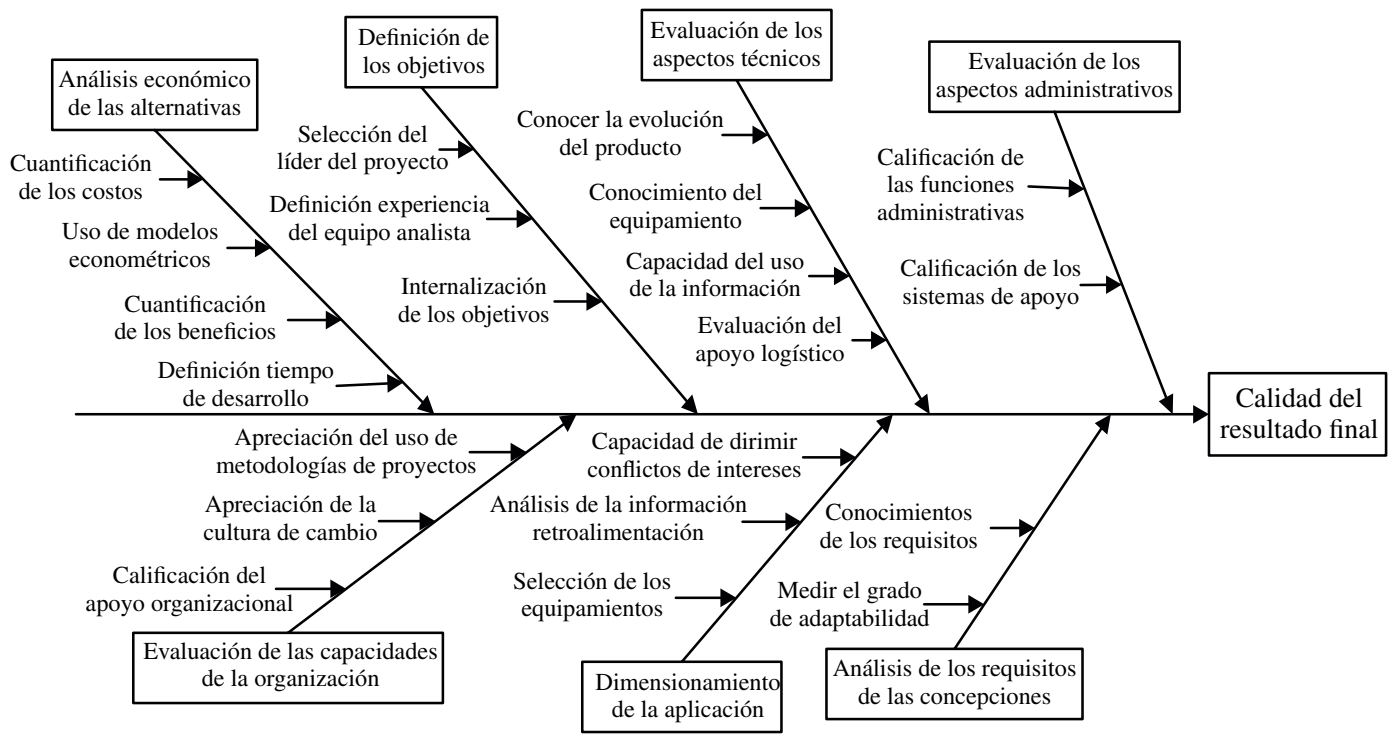

Figura 3. Diagrama de causa y efecto de los factores identificados que pueden ser fuente de riesgos. 
competencia para entender y evaluar abordajes y resultados de los análisis hechos, y finalmente para el equipo de analistas, es muy importante entender la cultura y valores de la organización.

Durante el proceso de estudio de alternativas para la innovación tener un ciclo de retroalimentación efectivo, o sea con información actualizada de indicadores de eficiencia, es fundamental porque a través de revisiones sucesivas, y a medida que se va adquiriendo un conocimiento más profundo durante el proceso de innovación, se pueden visualizar soluciones para cada riesgo posible en la futura aplicación del proyecto o bien debilidades en el proceso.

\section{DECISIONES PARA MINIMIZAR EL RIESGO}

Determinar, entender y administrar el riesgo es ahora una parte clave de cualquier política de mantenimiento o proceso de toma de decisiones, donde la determinación del riesgo es a menudo un proceso integral para satisfacer la legislación, alcanzar las marcas de clase mundial o al menos para implementar buenas prácticas en la gestión [11].

El riesgo es la combinación de la probabilidad de que un escenario no deseado en particular sea realidad, con las consecuencias o impactos negativos que produciría esa falla en el proceso de interés [13]. Es este par de parámetros los que deben ser tratados con el mayor cuidado por el equipo de análisis utilizando una metodología que los conduzca a la cuantificación de ellos, basados en una apreciación cualitativa y que esta pueda ser trasladada a un valor de referencia, el cual más adelante servirá para jerarquizar las acciones mitigantes del riesgo.

En cualquier situación que se exige una decisión están involucradas amenazas y oportunidades y ambas deben ser manejadas. Los cursos de acción están frecuentemente disponibles, los cuales reducen o neutralizan amenazas potenciales y ofrecen simultáneamente oportunidades para mejorías positivas en el desempeño del proyecto. No es aconsejable concentrarse en reducir amenazas sin considerar las oportunidades asociadas, como también no es aconsejable perseguir oportunidades sin consideración de las amenazas asociadas [14].

Para la valoración del par de parámetros que conforman el riesgo (probabilidad e impacto), se hace necesario que en el equipo de análisis estén presentes profesionales que tengan experiencia en proyectos de innovación similares, ya sea de la misma naturaleza o magnitud, a fin de poder definir, basados en su experiencia, cada uno de los factores que componen los parámetros conjuntamente con su relevancia (Figura 4). Esto último se ve reflejado en
Con respecto al nivel actual del factor evaluado, el riesgo para la característica se califica con valor:

5: Muy alta probabilidad de fracaso

4: Alta probabilidad de fracaso

3: Probabilidad media para fracasar

2: Probabilidad baja de fracasar

1: Probabilidad casi nula de fracasar

\begin{tabular}{|l|c|c|}
\hline Características & Ponderación & Calificación \\
\hline $\begin{array}{l}\text { Preparación } \\
\text { profesional }\end{array}$ & $30 \%$ & 3 \\
\hline $\begin{array}{l}\text { Gestión de } \\
\text { activos }\end{array}$ & $30 \%$ & 2 \\
\hline $\begin{array}{l}\text { Manejo de } \\
\text { personal }\end{array}$ & $40 \%$ & 4 \\
\hline $\begin{array}{l}\text { Calificación de la probabilidad } \\
\text { de riesgo }=\end{array}$ & 3,1 \\
\hline
\end{tabular}
Figura 4. Planilla de valoración de la probabilidad y del impacto.

En caso de producirse el fracaso a causa del factor, el impacto se califica como: 5: Muy alto con efecto irreversible 4: Alto, retrasará mucho al proyecto 3: Medio, significa sólo inversiones extras 2: Bajo, reacondicionamiento de recursos 1: Casi nulo, pequeños inconvenientes

\begin{tabular}{|c|c|}
\hline Calificación del impacto $=$ & 3 \\
\hline $\begin{array}{c}\text { Calificación final (probabilidad x } \\
\text { impacto) }\end{array}$ & 9,3 \\
\hline $\begin{array}{c}\text { Situación con inestabilidad } \\
\text { (ver explicación gráfica en Figura 5) }\end{array}$ & \\
\cline { 2 - 2 } & \\
\hline
\end{tabular}

\section{Situación con inestabilidad} Calificacion
de riesgo $=$ 
la ponderación que se define para cada componente que conforma el parámetro. Las ponderaciones y los valores que se ingresan para cada componente del parámetro son apreciaciones subjetivas del equipo de analistas, las cuales tienen que concordar con la realidad de cada situación bajo estudio.

Estas características pueden ser ponderadas según sea la importancia de poseer un cierto atributo para asegurar un nivel de éxito en la ejecución de las tareas del proyecto. En el ejemplo desarrollado en la Figura 4, para el caso relacionado con la designación del líder que tendrá a su cargo la implementación del proyecto, el equipo de analistas puede indicar que la característica de poseer un conocimiento acabado del problema es más importante que poseer ascendencia sobre sus colaboradores, por lo cual deberá tener una mayor ponderación en el momento de evaluar el nivel de probabilidad de riesgo. Para evaluar el impacto sobre el proyecto se debe tener en mente el efecto que provocaría en el proyecto si se da el fracaso de la tarea, por causa de la característica que está siendo evaluada.

Para las distintas combinaciones que se pueden dar para el par de parámetros, probabilidad e impacto, el espacio de implementación de mejorías se mueve principalmente en el eje de las probabilidades porque permite crear las condiciones para que la probabilidad asociada al riesgo disminuya, ya que ahí se pueden identificar acciones concretas y directas. Mientras que las acciones para mitigar el impacto no son inmediatas y se refieren principalmente a tener planes alternativos de contingencia, o bien crear defensas para que no se propague el daño, en el caso que sucedan trastornos en el proyecto, el cual es un escenario a futuro (Figura 5).

En [15] se identifican cuatro condicionantes para asegurar que la implementación de la innovación tenga éxito y se consiga cumplir con las metas trazadas. Estas condicionantes en el ámbito del mantenimiento son:

- El contenido estratégico se refiere a cómo y por qué la innovación es iniciada. Las acciones emprendidas deben ser consistentes con los objetivos globales de la empresa, por lo cual el eje central para la nueva iniciativa debe estar claramente identificado, como así también la participación activa de todos los niveles administrativos de la empresa.

- El contexto estratégico se puede dividir en dos: el contexto externo y el contexto interno. El contexto externo está directamente relacionado con la variabilidad del ambiente donde está inserta la organización productiva y son estos cambios los que repercuten en la función

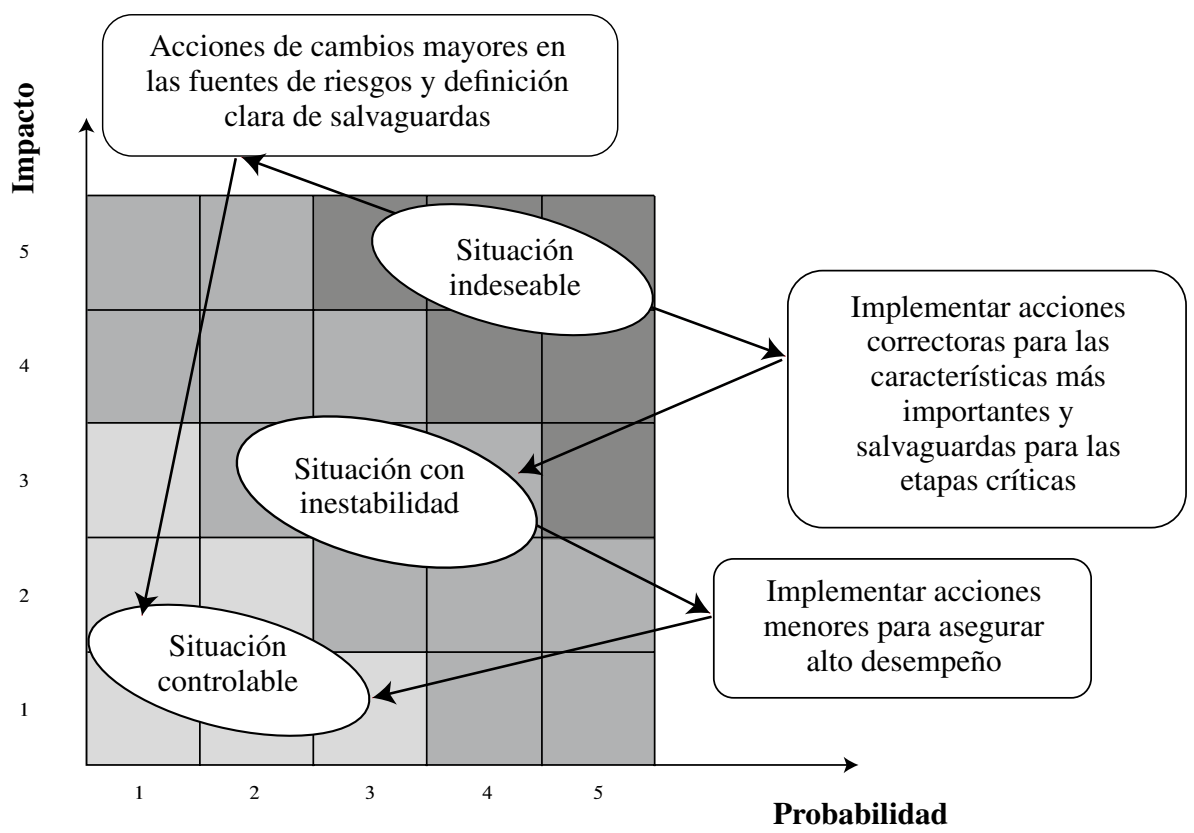

Figura 5. Plano de evaluación del par probabilidad e impacto y característica de las acciones correctoras. 
mantenimiento. Todo esto impone nuevas formas de gestión para los activos físicos. En referencia al contexto interno, las acciones tienen que llevar en cuenta los aspectos relacionados con la estructura organizacional y el proceso de toma de decisiones. También se deben incluir en el análisis la cultura organizacional y el aspecto del liderazgo.

- En el proceso operacional los aspectos las acciones tienen relación directa con la preparación y el planeamiento de la implementación de las actividades consideradas para el proyecto. Además de la coordinación de las diferentes áreas que participarán del proyecto y la aprobación de un cronograma de actividades, principalmente.

- El último punto considerado es la salida deseada del proyecto, que puede ser tangible o intangible. Para el equipo que está a cargo de la implementación del proyecto, debe tener la capacidad de analizar los resultados que se obtuvieron, sean estos exitosos o no. Para ambos casos, se deben analizar las fuentes de errores y las áreas dentro del planeamiento y de la ejecución, que pueden ser mejoradas y para donde estos conocimientos pueden ser transmitidos

Una herramienta muy usada para definir prioridades, contrastar situaciones y evaluar alternativas es el análisis matricial [8, 16-17]. Esta herramienta será usada en este trabajo para desarrollar el modelo que ayudará al administrador en su proceso de definición de las prioridades para implementar los mejoramientos necesarios en su sistema de mantenimiento (Figura 6). Además como existen restricciones de recursos y de compatibilidad entre las acciones posibles de ser implementadas, el análisis matricial es complementado con un modelo de programación lineal.

La matriz que se usa está compuesta, en un eje, por las acciones o actividades de mejoramiento que se podrían implementar para disminuir el riesgo y en el otro todos los factores que pueden ser fuentes potenciales de riesgos en el desarrollo del proyecto. Como los factores no son independientes entre sí, las acciones de mejoramiento pueden tener impacto en más de uno de ellos, por tanto ese efecto debe ser considerado al momento de definir cuál será en definitiva el conjunto de tareas que se implementarán, como también debe ser considerada en la jerarquización de las acciones la variación esperada en la calificación de cada característica. Todo esto da más de una dimensión a la selección de las acciones, con lo cual la aplicación de ellas será más eficiente.

La forma recomendada para usar la matriz es comenzar por definir las actividades que son necesarias implementar para pasar de una situación con inestabilidad a una situación más controlable, si es el caso, y como tienen relación directa con la disminución de la probabilidad de riesgo se evalúa el impacto con nota cinco. La definición de las acciones debe emerger del análisis que se realiza al momento de evaluar el riesgo asociado al factor, basado en sus características presentes.

A continuación se analiza cuál sería el impacto de la actividad $X j$ definida en el resto de los factores que identifican las fuentes de riesgo y calificar con nota cinco si el impacto es muy importante, con tres si el impacto es notorio, uno si aporta algún beneficio y nota cero si no hay relación alguna, tal como se muestra en el ejemplo reducido de la Figura 6. Este proceso dará una visión de las relaciones que existen entre los factores y las acciones de mejoramiento que se seleccionen.

La evaluación final del impacto de cada actividad $X j$ (Función G1) se determina según la ecuación (1):

$$
I T_{j}=\sum_{i} I_{i, j} P_{k} \quad \forall k
$$

donde:

$I T_{j}=$ impacto total de la actividad $j$.

$I_{i, j}=$ valor del impacto sobre el factor $i$ de la actividad $j$.

$P_{k}=$ ponderador del grupo $k$ del conjunto de factores.

En la valoración del aporte al mejoramiento esperado de cada actividad $X_{j}$ (Función G2) se hace uso de la ecuación (2):

$$
A T_{j}=\sum_{i}\left(\frac{I_{i, j} M_{i}}{\sum_{j} I_{i, j}}\right) P_{k} \quad \forall k
$$

donde:

$A T_{j}=$ aporte total al mejoramiento esperado de la actividad $j$.

$I_{i, j}=$ valor del impacto sobre el factor $i$ de la actividad $j$. 
$P_{k}=$ ponderador del grupo $k$ de los factores.

$M_{i}=$ mejoramiento esperado del factor $i$ por el grupo de acciones $j$.

Se ingresan además datos adicionales como ser el costo de la implementación de la actividad, el tiempo estimado de duración para conseguir el resultado deseado y las relaciones de interdependencia entre las acciones de mejoramiento (parte superior de la matriz). Estas últimas se identificarán por la siguiente notación: "o" acción que debe ser implementada, "d": acción que para ser implementada depende de la implementación de otra, "i": acción independiente de las restantes, y "e": acción que excluye a otra (Figura 6). Todo este conjunto de datos ingresados en la matriz de impacto darán los antecedentes para plantear un modelo de programación lineal (ecuaciones (3)), que permitirá optimizar el impacto total de las acciones de mejorías (Función G1) y el impacto global sobre la disminución esperada del riesgo (Función G2) sujetas a la disponibilidad de recursos (US\$ 7000) y relaciones de interdependencia.

Al igual como se planteó en la planilla de valoración de la probabilidad y del impacto también se puede dar peso relativo a cada grupo de factores que son fuentes de riesgos en la implementación del proceso de innovación. Para el caso desarrollado en la Figura 6 de dio mayor peso al aspecto económico en comparación con el resto de los grupos identificados. La definición del ponderador también es una decisión del grupo de análisis quienes decidirán si existen aspectos más relevantes que otros y que deberían ser atendidos con mayor premura.
Las acciones $X_{4}, X_{5}$ y $X_{8}$ no se implementarían de acuerdo a las condiciones definidas por el grupo a cargo del proceso de innovación. Este resultado debe ser corroborado con un análisis de tipo cualitativo basado en experiencias de especialistas en la materia y que es difícil modelar. Además, el modelo puede ser incrementado con otros tipos de restricciones, si la situación así lo indica, como ser tiempo disponible para la implementación (acciones de mejoramiento realizadas en serie o en paralelo), condicionantes de secuencia de implementación, entre otros.

Como la gestión del riesgo no es ampliamente usada y entendida, esto puede ser una ventaja competitiva significativa para aquellos que implementan la gestión del riesgo en sus proyectos [18] y más aún si en ellos existen recursos y personas comprometidas con el futuro de su organización.

\section{CONCLUSIONES}

La gestión efectiva del riesgo es la más importante herramienta de gestión que un administrador puede emplear para aumentar la probabilidad de éxito del proyecto, pero es necesario que el equipo de personas a cargo tenga la capacidad para hacer uso eficiente de las herramientas relacionadas con la administración de proyectos, análisis de escenarios y modelamiento matemático, como también disponer de un sistema de información actualizado, un líder con ascendencia y medidas de control aceptadas por los involucrados en el proyecto.

$$
\begin{array}{ll}
\operatorname{Max}_{1} \quad 2,7 X_{1}+5,8 X_{2}+5,45 X_{3}+2,8 X_{4}+4,45 X_{5}+3,15 X_{6}+4,5 X_{7}+2,1 X_{8} \\
\text { Max } & 1,08 X_{1}+1,96 X_{2}+1,81 X_{3}+0,53 X_{4}+1,22 X_{5}+0,78 X_{6}+0,67 X_{7}+0,42 X_{8} \\
\text { s.a. } \quad & 2000 X_{1}+600 X_{2}+1600 X_{3}+1200 X_{4}+1600 X_{5}+2000 X_{6}+800 X_{7}+600 X_{8} \leq 7000 \\
& X_{7}=1 \\
& X_{1}+X_{3}=2 \\
& X_{2}+X_{6}=2 \\
& X_{4}+X_{7}=1 \\
& X_{1}, X_{2}, X_{3}, X_{4}, X_{5}, X_{6}, X_{7}, X_{8}=\{0,1\}
\end{array}
$$

Solución: Función Objetivo $G_{1}=21,6$

Solución: Función Objetivo $G_{2}=6,3$

$X_{1}=1 ; X_{2}=1 ; X_{3}=1 ; X_{4}=0 ; X_{5}=0 ; X_{6}=1 ; X_{7}=1 ; X_{8}=0$ 


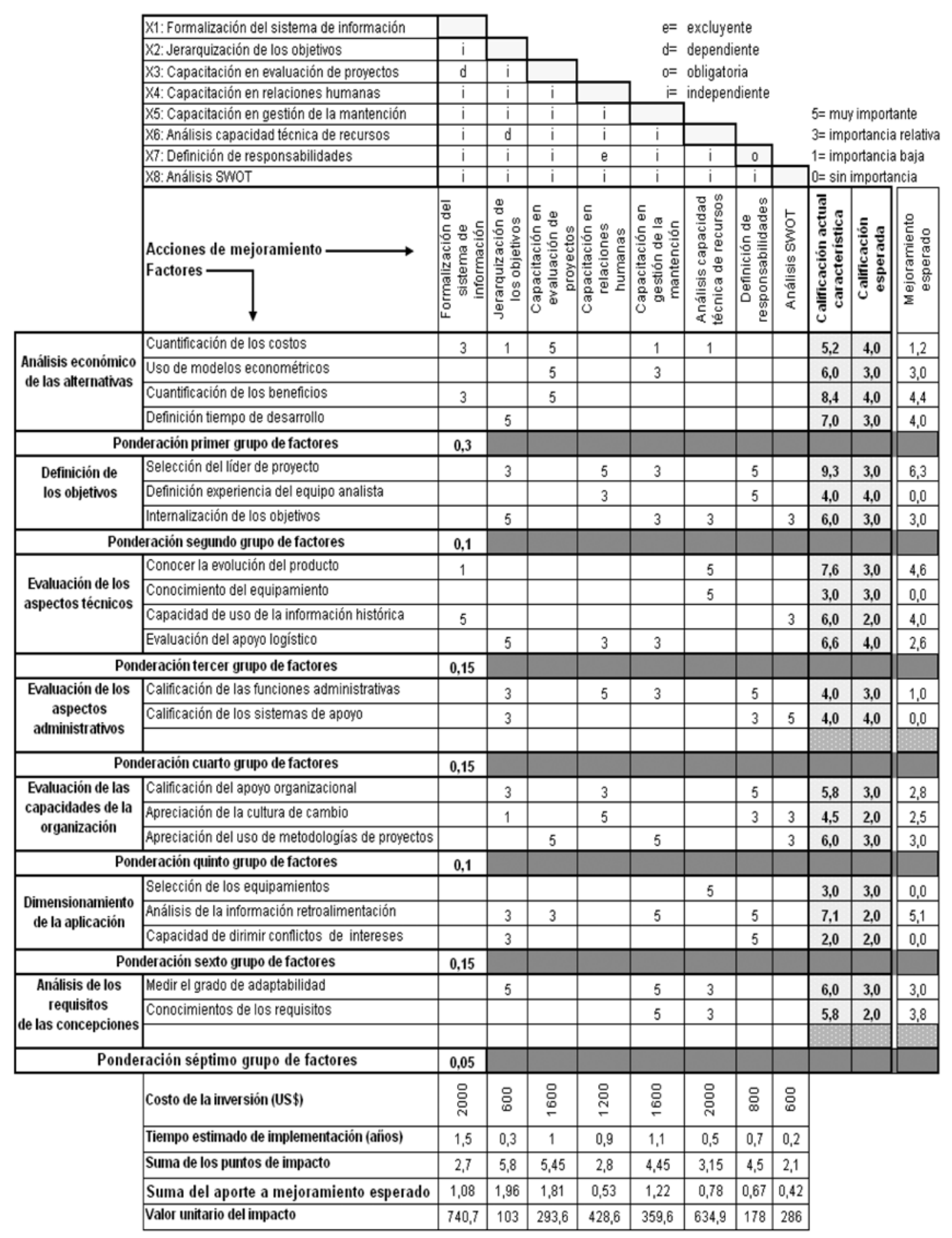

Figura 6. Matriz de relaciones entre acciones de mejoramiento y fuentes de riesgos. 
Se desarrolló en este trabajo una novedosa herramienta que permite jerarquizar las acciones de mejoramiento de las condiciones actuales de la organización, que se ve enfrentada a un proceso de innovación, basado en una evaluación de los factores fuentes de riesgos y la definición de las acciones que podrían minimizar esos riesgos, pero hay que ser acucioso en la definición del alcance de cada acción de mejoramiento para no introducir nuevas fuentes de riesgos en el desarrollo del proyecto. Por eso es que es importante el trabajo en equipo y conocer las condiciones del entorno de la organización.

La finalidad de la herramienta es ser un apoyo para la evaluación del riesgo inherente en la implementación de acciones de innovación y definición de acciones mitigantes, y su principal fuente de información es la apreciación del especialista o grupo de análisis y diseño sobre las condicionantes que podrían influir en la probabilidad de ser real un escenario no deseado.

Con el gráfico y planillas presentadas rápidamente se puede tener un valor bastante aproximado del riesgo, sin tener que entrar en cálculos probabilísticos. Nadie mejor para esto que aquella persona que conoce bien su ambiente y analiza futuras tareas de mejoramiento.

Este trabajo fue desarrollado para llenar un vacío en la gestión del mantenimiento, específicamente cuando se enfrentan tareas nuevas, pero eso no limita que su aplicación pueda ser extendida a otras áreas de la gestión industrial, definiendo los alcances y marco de referencia.

\section{REFERENCIAS}

[1] L. Tchankova. "Risk identification-basic stage in risk management". Environmental Management and Health. Vol. 13, Issue 3, pp. 290-297. 2002.

[2] J. Reason. "Managing the Risks of Organizational Accidents". Asgate, Burlington, USA. 1997.

[3] H. Kumamoto and E. Henley. "Probabilistic Risk Assessment and Management for Engineers and Scientists". IEEE Press. Second Edition. New York, USA. 1996.

[4] A. Dias, L. Calil, R. Rolim, R. Guembarovski and V. Cardoso. "Metodologia para gerenciamento de risco". IV Simposio
Internacional de Confiabilidade. São Salvador, BA. 2006.

[5] A. Mosleh, A. Dias, G. Eghhali and K. Fazen. "An Integrated Framework for Identification, Classification and Assessment of Aviation Systems Hazards". International Conference on Probabilistic Safety Assessment and Management (PSAM 7 and ESREL 04). Berlim, Germany. Ref. H11, p. 34. June, 2004. URL: http://www. psam7.org/finalprogram.pdf.

[6] A. Mobey and D. Parker. "Risk evaluation and its importance to project implementation". Work Study. Vol. 51, Issue 4, pp. 202-206. 2002.

[7] F. Espinosa. "Metodologia para Inovação da Gestão de Manutenção Industrial". Tese Doutorado. Universidade Federal de Santa Catarina. Brasil. 2006.

[8] A. Clarke and J. Garside. "The development of a best practice model for change management". European Management Journal. Vol. 15, Issue 5. 1997.

[9] T. Aven and J. Kørte. "On the use of risk and decision analysis to support decisionmaking". Reliability Engineering and System Safety. No 70. 2003.

[10] A. Rentes. "TransMeth-Proposta de uma Metodologia para Condução de Processos de Transformação de Empresas". Universidade de São Paulo. Escola de Engenharia de São Carlos. 2000.

[11] B. Hudson. "Risk-based maintenance". Petroleum Technology Quarterly. PTQ Q4, pp. 37-45. 2006.

[12] F. Backlund and J. Hannu. "Can we make maintenance decision on risk analysis results?". Journal of Quality in Maintenance Engineering. Vol. 8, Issue 1. 2002.

[13] J. Emblemsvåg and L. Kjølstad. "Strategic risk analysis-a field version". Management Decision. Vol. 40, Issue 9, pp. 842-852. 2002.

[14] S. Ward and C. Chapman. "Transforming project risk management into project uncertainty management". International Journal of Project Management. Vol. 21, pp. 97-105. 2003.

[15] F. Okumus. "A framework to implement strategies in organizations". Management Decision. Vol. 41, Issue 9, pp. 871-882. 2003. 
[16] C. Govers. "What and how about quality function deployment (QFD)". Int. J. Production Economics. Vol. 46-47, pp. 575-585. 1996.

[17] S. Han, S. Chen, M. Ebrahimpour and M. Sodhi. "A conceptual QFD planning model". International Journal of Quality \& Reliability
Management. Vol. 18, Issue 8, pp. 796-812. 2001.

[18] Y. Kwak and J. Stoddard. "Project risk management: lessons learned from software development environment". Technovation. Vol. 24. 2004. 